\title{
AUTOMATION OF CAPACITY BIDDING WITH AN AGGREGATOR USING OPEN AUTOMATED DEMAND RESPONSE
}

Prepared For:

California Energy Commission

Prepared By:

Lawrence Berkeley National Laboratory

Demand Response Research Center

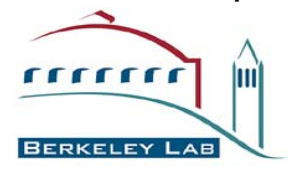

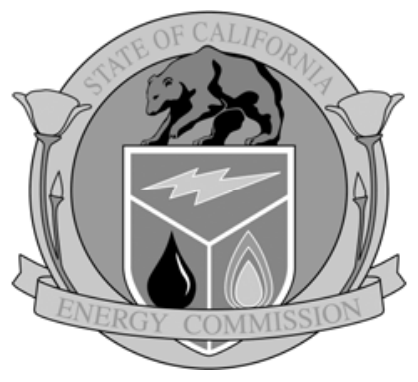

Arnold Schmarzenegger Governor

5
8
$\frac{5}{4}$
$\frac{5}{8}$
$\frac{8}{2}$
$\frac{1}{2}$
$\frac{1}{2}$
$\frac{1}{2}$ 

Prepared by:

Lawrence Berkeley National Laboratory

Demand Response Research Center

Sila Kiliccote

Mary Ann Piette

Berkeley, CA

Contract No. 500-03-026

Prepared For:

Public Interest Energy Research (PIER) California Energy Commission

\author{
Kristy Chew
}

Contract Manager

Mike Gravely

Program Area Lead

Energy Systems Integration

Mike Gravely

Office Manager

Energy System Research Office

Martha Krebs

PIER Director

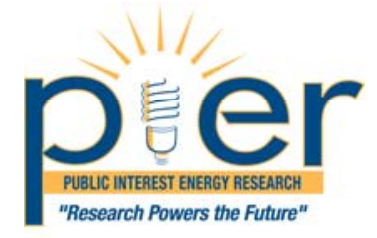

Thom Kelly

Deputy Director

ENERGY RESEARCH \& DEVELOPMENT DIVISION

Melissa Jones

Executive Director

\title{
DISCLAIMER
}

This report was prepared as the result of work sponsored by the California Energy Commission. It does not necessarily represent the views of the Energy Commission, its employees or the State of California. The Energy Commission, the State of California, its employees, contractors and subcontractors make no warrant, express or implied, and assume no legal liability for the information in this report; nor does any party represent that the uses of this information will not infringe upon privately owned rights. This report has not been approved or disapproved by the California Energy Commission nor has the California Energy Commission passed upon the accuracy or adequacy of the information in this report. 


\section{Disclaimer}

This document was prepared as an account of work sponsored by the United States Government. While this document is believed to contain correct information, neither the United States Government nor any agency thereof, nor The Regents of the University of California, nor any of their employees, makes any warranty, express or implied, or assumes any legal responsibility for the accuracy, completeness, or usefulness of any information, apparatus, product, or process disclosed, or represents that its use would not infringe privately owned rights. Reference herein to any specific commercial product, process, or service by its trade name, trademark, manufacturer, or otherwise, does not necessarily constitute or imply its endorsement, recommendation, or favoring by the United States Government or any agency thereof, or The Regents of the University of California. The views and opinions of authors expressed herein do not necessarily state or reflect those of the United States Government or any agency thereof or The Regents of the University of California. 


\section{Acknowledgements}

The work described in this report was funded by the Emerging Technologies and Technical Audit /Technology Incentives program at San Diego Gas \& Electric Company. Additional funding was provided by the Demand Response Research Center, which is funded by the California Energy Commission's (Energy Commission) Public Interest Energy Research (PIER) Program, under Work for Others Contract No. 500-03-026, Amendment \#1 and by the U.S. Department of Energy under Contract No. DE-AC02-05CH11231. The authors are grateful for the extensive support from numerous individuals who assisted in this project:

Kristy Chew and Martha Brook (California Energy Commission)

Julia Mendoza, Matt Bucur, Terry Mohn, and Austin D’Lima at SDG\&E for program management and technical assistance effort.

Peter Livingston (Livingston Engineering) for his assistance with the test event.

California Institute for Energy and the Environment for its assistance with the contract

Sean Nunes (Site Controls, LLC) for coordination of efforts, data sharing, and openness to collaborate.

Dan Hennage (Akuacom) for development of the DR Automation Server System

Please cite this report as follows:

Kiliccote, Sila, and Mary Ann Piette (Lawrence Berkeley National Laboratory). Automation Development and Testing of the Capacity Bidding Program for San Diego Gas and Electric Company. California Energy Commission, PIER Energy Systems Integration Program. CEC-500-2008-059. 


\section{Preface}

The California Energy Commission's Public Interest Energy Research (PIER) Program supports public interest energy research and development that will help improve the quality of life in California by bringing environmentally safe, affordable, and reliable energy services and products to the marketplace.

The PIER Program conducts public interest research, development, and demonstration (RD\&D) projects to benefit California.

The PIER Program strives to conduct the most promising public interest energy research by partnering with RD\&D entities, including individuals, businesses, utilities, and public or private research institutions.

PIER funding efforts are focused on the following RD\&D program areas:

- Buildings End-Use Energy Efficiency

- Energy Innovations Small Grants

- Energy-Related Environmental Research

- Energy Systems Integration

- Environmentally Preferred Advanced Generation

- Industrial/Agricultural/Water End-Use Energy Efficiency

- Renewable Energy Technologies

- Transportation

The Automation of Capacity Bidding With an Aggregator Using Open Automated Demand Response is a final report for the Demand Response Research Center (contract 500-2003-024) conducted by Lawrence Berkeley National Laboratory. The information from this project contributes to PIER's Energy Systems Integration Program.

For more information on the PIER Program, please visit the Commission's website at: http://www.energy.ca.gov/pier or contact the Energy Commission at 916-654-5164. 


\section{Table of Contents}

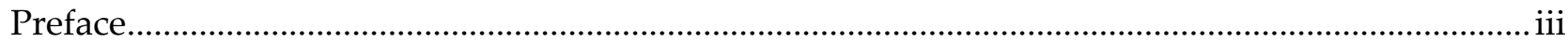

Abstract and Keywords............................................................................................................. vii

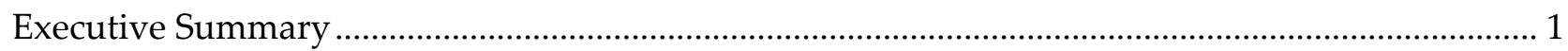

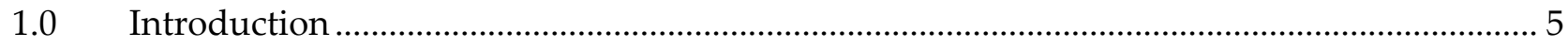

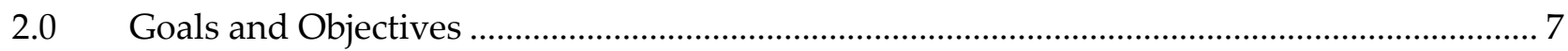

3.0 Open Automated Demand Response Communications Infrastructure................................ 9

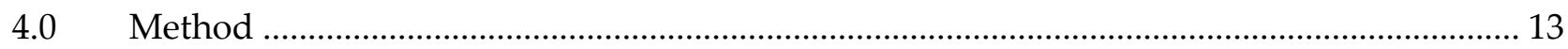

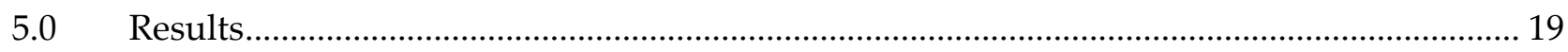

5.1 End-to-End Automation Test ................................................................................. 20

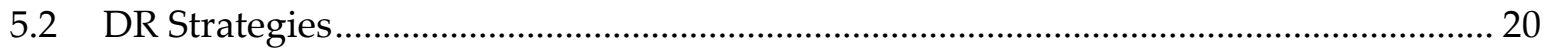

5.3 Demand Response Shed Analysis............................................................................. 21

5.4 Total Automated CBP Implementation Capacity ..................................................... 25

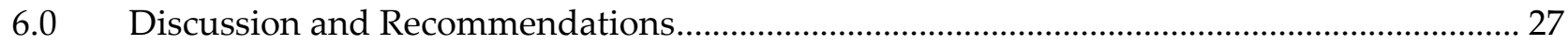

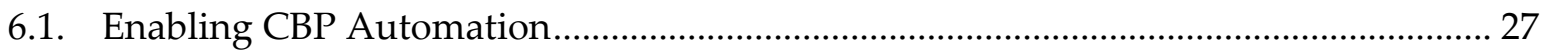

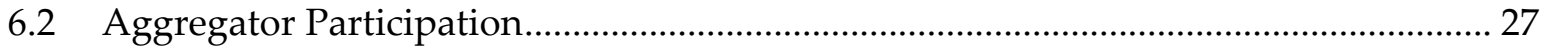

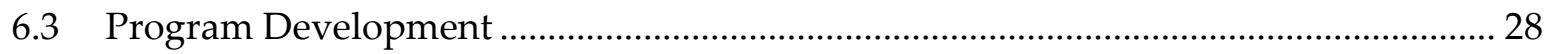

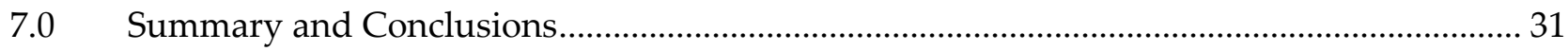

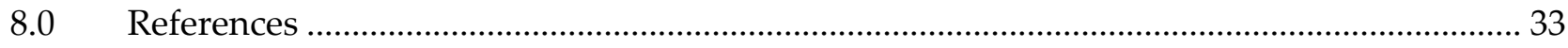

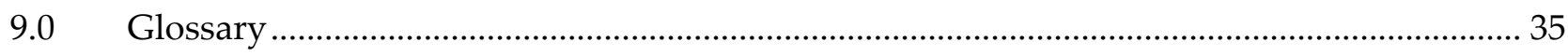

\section{List of Figures}

Figure 1 Generic Automated DR Open-Interface Standard Architecture ................................. 11

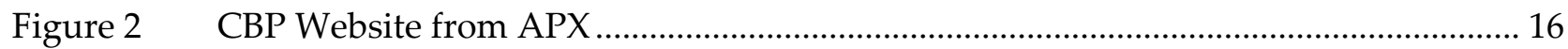

Figure 3 APX and DRAS Integration for CBP Automation .................................................... 19

Figure 4 Open Auto-DR and Proprietary CBP Automation as Implemented by Site Controls 21

Figure 5 Aggregated Load Profile of the Test on October 3, 2007 ......................................... 24

Figure $6 \quad$ Retail C3 Load Profile with Its Baseline................................................................... 25

\section{List of Tables}

Table $1 \quad$ History of Open Auto DR ......................................................................................... 10

Table 2 Load Reduction Incentives $(\$ / \mathrm{kW})$ for CBP Day-Ahead Program ............................. 165

Table $3 \quad$ Load Reduction Incentives $(\$ / \mathrm{kW})$ for CBP Day of Program ...................................... 15

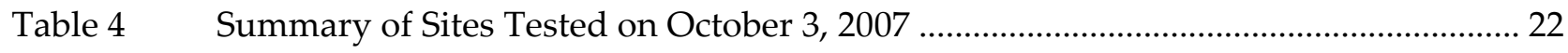

Table 5 Summary of Average Sheds for Each Site with Total Average Shed........................ 245

Appendix A Site Summary and October 3, 2007 Test Results 


\begin{abstract}
This report summarizes San Diego Gas \& Electric Company's collaboration with the Demand Response Research Center to develop and test automation capability for the Capacity Bidding Program in 2007. The report describes the Open Automated Demand Response architecture, summarizes the history of technology development and pilot studies. It also outlines the Capacity Bidding Program and technology being used by an aggregator that participated in this demand response program.

Due to delays, the program was not fully operational for summer 2007. However, a test event on October 3, 2007, showed that the project successfully achieved the objective to develop and demonstrate how an open, Web-based interoperable automated notification system for capacity bidding can be used by aggregators for demand response. The system was effective in initiating a fully automated demand response shed at the aggregated sites. This project also demonstrated how aggregators can integrate their demand response automation systems with San Diego Gas \& Electric Company's Demand Response Automation Server and capacity bidding program.
\end{abstract}

Keywords: Open automated demand response, automated capacity bidding program, demand response aggregator, demand response 


\section{Executive Summary}

In 2007, San Diego Gas \& Electric Company (SDG\&E) began offering demand response programs that allow aggregators to include customers with loads greater than 20 kilowatts $(\mathrm{kW})$. The SDG\&E Capacity Bidding Program is a reliability-based demand response program. ${ }^{1}$ SDG\&E collaborated with the Demand Response Research Center to develop and test automating the Capacity Bidding Program. The project's objectives were to:

- Develop and demonstrate how an open, Web-based interoperable. ${ }^{2}$ automated notification system for capacity bidding can be used by aggregators for demand response.

- Evaluate the effectiveness of such a system. Determine how aggregators can respond to this form of automation for Capacity Bidding Program.

- Evaluate what type of demand response strategies can be automated for Capacity Bidding Program for small commercial customers to provide effective demand response.

- Explore how automation of control strategies can increase participation rates and demand response.

To achieve the objectives, the research had four key elements: 1) interview aggregators to identify sites that can deliver end-to-end automation; 2) design and automate the Capacity Bidding Program by developing requirements and integration concepts; 3 ) test the automation among all the parties involved and; 4) field test at selected facilities that uses the same technology for the selected aggregator.

The Open Automated Demand Response (Open Auto-DR) architecture consists of two major elements built on open and interoperable standard communication model. First, a demand response automation server provides standard signals that notify electricity customers of demand response events. Second, a Demand Response Automation Server client is at the customer's site to listen and provide automation signals to existing pre-programmed controls. The Open Auto-DR architecture provides the following benefits:

- Open Standards-Provides a standardized demand response communication and signaling infrastructure using open, non-proprietary, industry-approved data models that can be implemented for both dynamic prices and demand response emergency or reliability events.

1. Demand response is a set of time-dependent program activities and tariffs designed to reduce electricity use or shift usage to another period. Demand response provides control systems that encourage load shedding or load shifting during times when the electric grid is near its capacity or electricity prices are high. Demand response helps to manage building electricity costs and to improve electric grid reliability.

2. Interoperable is defined as: different programs capable of exchanging data via a common set of exchange formats, to read and write the same file formats, and to use the same protocols. 
- Flexibility-Provides open communications interfaces and protocols that are flexible, platform-independent, interoperable, and transparent to end-to-end technologies and software systems.

- Innovation and Interoperability-Encourages open innovation and interoperability, and allows controls and communications within a facility or enterprise to build on existing strategies to reduce technology operation and maintenance costs, stranded assets, and obsolescence in technology.

- $\quad$ Ease of Integration-Promotes integration of common energy management and control systems (EMCS), centralized lighting, and other end-use devices that can receive a relay or Internet signals (such as XML)

- Remote Access - Facilitates opt-out/override functions through a customer Web portal to manage standardized demand response-related operation modes to demand response strategies and control systems.

- Customer System Stability-Standardizing the information model used to support price signals and reliability event information reduces the hardware and systems costs and provides the customer with the capability to easily participate in one or more demand response options to switch between options or service providers and to fully automate response strategies compatible with their individual requirements.

To guarantee end-to-end automation with the aggregators in the automation loop, aggregators participating in Capacity Bidding Program with full automation must have automated systems that control each customer's end-use equipment.

The results of the Capacity Bidding Program automation implementation show that:

- The project developed and demonstrated an open, Web-based interoperable automated notification system that was used by aggregators for capacity bidding.

- The system was effective in the initial field tests, although the late project start did not allow for evaluation of all of the aggregator's customer sites.

- Aggregators that receive demand response automation signals, process them, and send proprietary signals to their customers' control points without a human in the loop are considered "end-to-end fully automated." Some aggregators use full end-to-end automation while others are working towards it. SDG\&E is making additional automation funds (extra \$50/kilowatt [kW]) available when an aggregator demonstrates automation. Therefore, aggregators can participate in the Capacity Bidding Program with full automation today and increased participation is expected in the coming years. Proprietary automated demand response systems are not fully Open Auto-DR based systems.

- The auto-demand response concept previously developed for price responsive programs was easily expanded to enable reliability programs such as the Capacity Bidding Program. The time and cost associated with the development of automated programs depends on the level of integration between the utility information systems and the demand response automation server. 
- Aggregator involvement frees the utility from burdens such as recruitment and technical assistance throughout the automation installation and commissioning.

- Under the current structure of SDG\&E's Technical Audit/Technology Incentives program, verification of automation can easily be integrated with load shed reduction tests and can be overseen by the test engineers.

\section{Benefits to California}

There are many benefits to California consumers of a standardized demand response communication and signaling infrastructure using open, non-proprietary, industryapproved data models that can be implemented for both dynamic prices and demand response emergency or reliability events. First, it encourages open innovation and interoperability, and allows controls and communications in a facility or enterprise to build on existing strategies that reduce technology operation and maintenance costs, stranded assets, and obsolesce in technology. Second, it provides open communications interfaces and protocols that are flexible, platform-independent, interoperable, and transparent to endto-end technologies and software systems and increases the transparency of demand response technologies. Finally, standardizing the information model used to support price signals and reliability event information provides the customer with the capability to easily participate in one or more demand response options to switch between options or service providers and to fully automate response strategies compatible with their individual requirements. In addition, by working with an aggregator who develops demand-side management technologies for small commercial market segment, Open Auto-DR with an aggregator reaches out to a new market segment in California. 


\subsection{Introduction}

Demand response (DR) is the process of managing energy use dynamically through cooperation between power customers, their electric utility, and the electric system's operator (the independent system operator, or ISO). When the electrical grid is near capacity-for example, when too many air conditioners start laboring on a hot summer day - the utilities inform power consumers that there is a problem. In response, power consumers who have signed up for a utility's DR program act to reduce their nonessential needs - for example, by cutting down on the lighting and cooling in underused parts of their buildings. This helps avoid a grid crisis and benefits consumers through lower energy bills, since the peak power consumed during highuse periods is the most expensive power.

In 2007, the San Diego Gas \& Electric Company (SDG\&E) began offering DR programs that allow aggregators to include customers with loads greater than 20 kilowatts $(\mathrm{kW})$ that have interval power meters. The SDG\&E Capacity Bidding Program (CBP) is a reliability-based DR program. ${ }^{3}$ Most customers participate in the SDG\&E CBP through an aggregator (see Section 6). Aggregators combine the demand response activities of numerous customers and they deliver DR services and technologies that help customers participate and respond to DR events. This document reports on the first full-scale test that uses the Open Auto-DR systems with aggregators. Aggregators provide a significant portion of the DR resources in California. The purpose of fully automated DR, of which CBP automation with SDG\&E is one example, is to improve the responsiveness and participation of electricity customers in DR events and to lower overall costs for DR.

SDG\&E collaborated with the Demand Response Research Center (DRRC) (managed by Lawrence Berkeley National Laboratory, LBNL) to develop and test automating CBP. Levels of automation of DR can be defined as follows.

- Manual Demand Response involves a labor-intensive approach such as manually turning off or changing comfort set points at each equipment switch or controller.

- Semi-Automated Demand Response involves a pre-programmed demand response strategy initiated by a person via a centralized control system.

- Fully-Automated Demand Response does not involve human intervention, but is initiated at a home, building, or facility through receipt of an external communications signal.

The receipt of the external signal initiates pre-programmed demand response strategies. The authors of this report refer to this as Auto-DR (Piette et al. 2005). One important feature in Auto-DR is that a homeowner or facility manager can "opt out" or "override" a voluntary DR event if the event comes at a time when a reduction in end-use services is not acceptable.

3. More information on SDG\&E's Capacity Bidding program is available at http://www.sdge.com/esc/largecb.shtml 
From the customer side, modifications to the site's electric load shape can be achieved by modifying end-use loads. Examples of demand response strategies include reducing electric loads by dimming or turning off non-critical lights, changing comfort thermostat setpoints, or turning off non-critical equipment. These demand response strategies are triggered by specific actions set by the electricity service provider. Many electricity customers have suggested that automation will help them institutionalize their demand response.

This report focuses on and discusses the specific results of the CBP automation tests that LBNL conducted during 2007 with SDG\&E. The structure of this report is as follows.

- Section 2, Project Objectives, provides a discussion of the project objectives.

- Section 3, Automated Demand Response Communications Infrastructure describes the key features of automated DR systems and a brief history of the technology development and demonstrations during the last five years.

- Section 4, Methodology, outlines the project methodology covering the technology used for the automation plus the CB program design and steps for participation. Section 3 also has the evaluation methods used in the study. These include the peak demand baseline models, data collection methods and evaluation of effectiveness of automation.

- Section 5, Results, provides an overview of the aggregated and individual facility demand reductions during the test events and summarizes an aggregator's (Site Control) participation in 2007.

- Section 6, Discussions and Recommendations is a discussion of key findings relative to the project objectives and future directions of the automation of CBP.

- Appendices provide summary of sites and DR strategies and results from the test conducted on October 3, 2007. 


\subsection{Goals and Objectives}

The overall goal of this project is to understand and evaluate methods to improve demand response (DR) effectiveness in California. One major element of this evaluation has been automation technology. Specific objectives of this project were to:

- Develop and demonstrate how an open, Web-based interoperable automated notification system for capacity bidding can be used by aggregators for demand response.

- Evaluate the effectiveness of such a system.

- Determine how aggregators can integrate their DR automation systems with the DR Automation Server to support a capacity bidding program (CBP).

- Develop a method to characterize aggregator's automation systems.

- Identify effective small commercial DR strategies. 


\subsection{Open Automated Demand Response Communications Infrastructure}

Open Automated Demand Response (Open Auto-DR) for commercial and industrial facilities can be defined as fully automated demand response (DR) initiated by a standard signal from a utility or other appropriate entity and provide fully-automated connectivity to customer enduse control strategies. Open Auto-DR uses utility provided price, reliability, or event signals linked to energy management control systems (EMCS) ${ }^{4}$ or other control equipment to automatically initiate customer pre-programmed energy management strategies. Key features of Open Auto-DR include (Piette et al. 2007):

- Signaling - Open AutoDR-based technology should provide continuous, secure, reliable, two-way communication with end-use customers to allow end-use sites to be identified as listening and acknowledging receipt of DR signals.

- Open Industry Standards - Open AutoDR consists of open, interoperable industry standard control and communications technologies designed to integrate with both common energy management and control systems and other end-use devices that can receive a dry contact relay or similar signals (such as internet based XML).

- Timing of Notification - Day ahead and day of signals are provided by Open AutoDR technologies and systems to facilitate a diverse set of end-use strategies such as building pre-cooling for "day ahead" notification, or near real-time communications to implement "day of" control strategies. Timing of a DR automation server (DRAS) communications must consider day-ahead events that include weekends and holidays

A Technical Advisory Group (TAG) managed by the Demand Response Research Center has been formed to formalize the Open Auto-DR standard which is being developed to support open, interoperable DR automation. The TAG is made up of three utilities (SDG\&E, Pacific Gas and Electric Company [PG\&E], and Southern California Edison [SCE]), research organizations (Electric Power Research Institute, etc.), and standards organizations such as National Institute of Standards and Technologies (NIST).

LBNL research has found that many building EMCS and related lighting and other controls can be pre-programmed to initiate and manage electric demand response. Table 1 summarizes the history of sites, server and client technologies, as well as utility support.

4. Energy management and control systems are centralized controls, generally with personal computer interface, primarily for heating, ventilation, and air conditioning systems. These systems sometimes also provide lighting control, as well as control of fire and life-safety systems. 
Table 1. History of Open Auto-DR

\begin{tabular}{|r|r|l|l|l|}
\hline Year & \# of Sites & DRAS & Site Communication & Utility \\
\hline \hline 2003 & 5 & Infotility & XML Gateway Software & None \\
\hline 2004 & 18 & Infotility & XML \& Internet Relay & None \\
\hline 2005 & 11 & Akuacom & XML \& Internet Relay & PG\&E \\
\hline 2006 & 25 & Akuacom & XML, Internet Relay, CLIR & PG\&E, SDG\&E \\
\hline
\end{tabular}

Source: Lawrence Berkeley National Laboratory

The 2003 automated DR technology development began with the design of a fictitious price signal and automation server that could represent price signals in Extensible Markup Language (XML) messages to support interoperable communications. Five facilities were recruited and each facility had Energy Information Systems (EIS) (Piette et. al. 2004), EMCS, technology gateways, and a variety of end-use load reduction strategies (Piette et. al. 2005). The test resulted in fully automated shedding during two events with an average peak reduction of about 10 percent. The 2004 design considered that many facilities may not have EIS or EMCS so a low-cost internet relay was used to communicate with on-site equipment. The average demand reduction for these 15 sites was 0.53 watts per square foot $\left(\mathrm{W} / \mathrm{ft}^{2}\right)$ or about 14 percent of the whole building electric-peak demand. The relay was used for two years but replaced because of internet security concerns. It was replaced with a communications device called a Client \& Logic with Integrated Relay (CLIR) box which is a self-configuring secure internet relay device.

In 2005 the research team began a formal collaboration with PG\&E to offer Open Auto-DR as part of the Critical Peak Pricing (CPP) Program. The DRRC worked with a software and hardware development company, Akuacom, to develop a new DR automation server (DRAS). For the eight sites that participated in the fully automated CPP (Auto-CPP) event on September 29,2005 , the average demand response ranged from 0 to 24 percent per site for the medium price period and 4 to 28 percent per site during the high price period, with an average of 9 percent and 14 percent overall for the two price periods. Following the pilot automated CPP test in 2005 the research team began a more formal partnership with PG\&E's Emerging Technologies Program. Among the 25 Auto-CPP sites, site responses to 125 events were fully automated and evaluated in this study. The average peak demand reduction was 14 percent of the whole-facility load based on the three-hour high-price period. (Piette et.al. 2006, 2007)

As mentioned, the purpose of fully automated DR, of which CBP automation with SDG\&E is one example, is to improve the responsiveness and participation of electricity customers in DR events and to lower overall costs for DR. Open Auto-DR involves systems that automatically reduce electric demand in facilities upon receipt of a signal denoting an electric grid emergency or a rise in the price of electricity. In CBP automation, aggregators bid into the program using an interface provided by the utility five business days before the start of each month. Bids that are within the program rules are accepted and the aggregators are notified. Automated dayahead and day-of signals, indicating a DR event are broadcast by a web services server through the Internet using the meta-language XML (eXtensible Markup Language). Each of the participating aggregators monitors this common signal using a web services client application. The aggregator automatically sends automated signals downstream to their customers' end-use 
controls to initiate sheds of site-specific electric loads when the DR event is dispatched. The system is designed to implement demand response without human intervention.

Open Auto-DR architecture, as displayed in Figure 1, consists of two major elements built on open-interface standards model. First, a Demand Response Automation Server (DRAS) provides signals that notify electricity customers of DR events. Second, a DRAS client is at the customer's site to listen and provide automation signals to existing pre-programmed controls. There are two types of DRAS clients:

1. A Client and Logic with Integrated Relay (CLIR) or simple client for legacy control systems.

2. A Web Services software or smart client for sophisticated control systems.

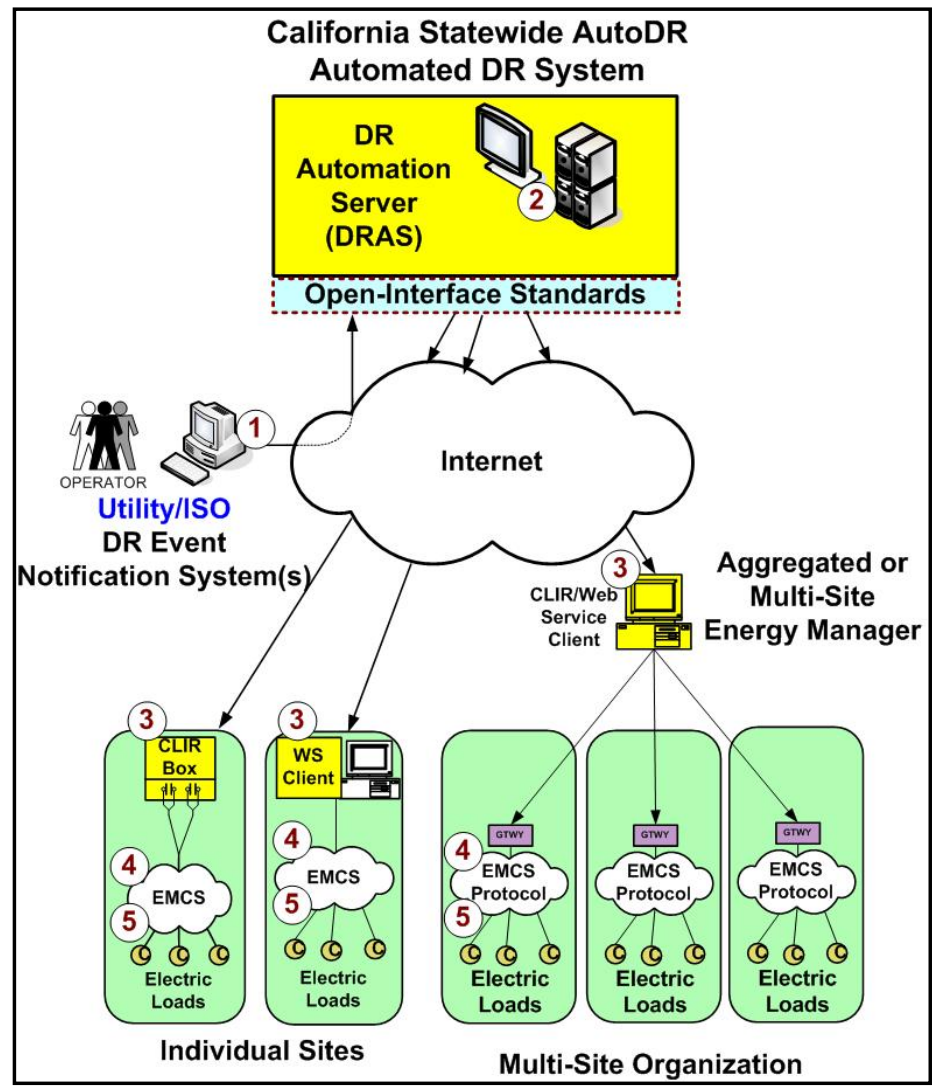

Figure 1. Generic Automated DR Open-Interface Standard Architecture

Source: Lawrence Berkeley National Laboratory

As shown in above figure, the steps involved in the Open Auto-DR process during a DR event are:

1. The Utility or ISO defined DR event and price/mode signals are sent to the DRAS. 
2. DR event and price services published on a DRAS.

3. DRAS Clients (CLIR or Web Service) request event data from the DRAS every minute. The aggregated systems in use in the SDG\&E test are further described below.

4. Customized pre-programmed DR strategies determine action based on event price/mode.

5. Facility EMCS or CLIR carries out load reduction based on DR event signals and strategies.

Open Auto-DR systems use an XML-based Web Service Oriented Architecture (SOA) for platform-independent, interoperable systems. Open Auto-DR systems have been used for CPP in previous programs during 2005, 2006, and 2007. The SDG\&E CBP automation implementation is a new addition to a set of already automated programs such as CPP and Demand Bidding Programs offered by PG\&E. The architecture for the SDG\&E CBP aggregator system is further described below. 


\subsection{Method}

From 2003 through 2006 the Demand Response Research Center (DRRC) developed and tested a series of demand response (DR) automation communications technologies known as Open Automated Demand Response (Open Auto-DR) (Piette et al, 2005a, 2005b, 2006, 2007). During 2006 the DRRC performed a pilot Automated Demand Response Study with the San Diego Gas and Electric (SDG\&E) Emerging Technologies Program. The participants included a Target retail store in Chula Vista and two Whole Foods Market stores in La Jolla and Hillcrest. Target was able to develop a web-services based software client in a few days similar to the Open Auto-DR systems they had previously used in Northern California and was able to participate in a test event. The Open Auto-DR design for Whole Foods Market involved the use of a Client and Integrated Relay Logic (CLIR) box to be directly connected to a) selected circuits at the lighting panel to switch off selected set of lights and b) heating, ventilation, and air conditioning (HVAC) controls to increase temperatures in the store upon receipt of a DR signal. The Whole Foods Market Open Auto-DR design was never installed within the timeframe of the project, but was useful in evaluating methods to automate DR in small commercial facilities. Appendix $B$ provides a graphic describing the various Open Auto-DR implementations with SDG\&E in 2006.

In 2007 LBNL/DRRC collaborated with SDG\&E to develop and test the automation of the Capacity Bidding Program (CBP). This report summarizes the design, deployment, and results from the 2007 CBP automation. The program was designed to evaluate the feasibility of deploying automation systems that allow aggregators to participate in CBP with a fullyautomated response. The methodology has four key elements:

1. Interviews with Aggregators to Identify Sites

Following SDG\&E's decision to develop and demonstrate Open Auto-DR with DR aggregators and the CBP, the DRRC began evaluating technologies the DR aggregators use. SDG\&E initially identified two aggregators within the CBP for the automation compatibility. Each aggregator worked with LBNL to identify sites within their portfolios and selected and implemented control strategies for demand response as well as developing automation system designs based on existing Internet connectivity and building control systems. While one of the aggregators was not able to identify any sites within their portfolio, the other (Site Controls) had fully automated all of their sites and so could participate in CBP with full automation. This aggregator could listen for and receive the signal sent by a demand response automation server (DRAS) and automatically pass on related signals through their proprietary network to equipment at the customer sites.

\section{Automated Capacity Bidding System Design}

SDG\&E works with a company called APX that collects bids for and notifies customers and aggregators prior to CBP events. APX works with all of the investor-owned utilities (IOUs) in California and has designed the on-line bidding portal for all the aggregators and customers participating in the CBP. In developing the automation systems for the CBP, Akuacom developed and maintained the DRAS. Development effort was very short since the work started mid-August 2007 and the CBP period ended on October 31, 2007. 
Therefore, instead of a fully integrated approach, the program was divided into bidding and event management tasks. While bids were accepted and settled by APX, Akuacom sent the DR event signals to the aggregator's system for each DR event (see Figure 3 below).

3. Automation Testing

A series of automation tests were developed to evaluate full end-to-end connectivity. The DRRC investigated the technology installed at each site to understand and document the networking infrastructure implemented by Site Controls. Together with a SDG\&E test engineer, the DRRC conducted communications tests to ensure that the DRAS correctly provided and logged the continuous communication of the CBP event signals with the site's EMCS. Procedures for communications tests are outlined in Appendix A. The DRRC also observed and evaluated DR shed strategies to ensure proper commissioning of controls. The communication system allowed sites to receive day-ahead as well as day-of signals for pre-cooling, a DR strategy used at some sites.

\section{DR Field Tests}

In order to evaluate the DR load reduction $(\mathrm{kW})$ from the automated systems, the DRRC conducted an analysis using several baseline models to estimate how much each building would use had the DR events not taken place (Coughlin et al., 2008). SDG\&E uses a standard baseline model that the CBP payments are based on. The CBP baseline model that LBNL reproduced is based on the site's electricity consumption from noon to 6 p.m. for the three days with highest consumption of the previous 10 non-weekend days; it is not normalized for weather. The second model evaluated by the DRRC used the CBP baseline model but with a morning adjustment. The morning adjustment is a multiplier calculated from the ratio of actual loads to the CBP baseline two hours prior to the DR event start time. The third model, the outside air temperature (OAT) regression baseline model, is based on OAT data and site electricity consumption from the previous 10 days. The OAT model uses weather regressions from the 15-minute electric load data during each event day (Coughlin et al., 2008). These baseline models were used to evaluate the demand reduction during each DR event for each site.

The Open Auto-DR signal was customized for SDG\&E's CBP. The program and the related signal design are as follows. The Capacity Bidding Program offers commercial and industrial customers monthly financial incentives that commit to reducing power by a pledged amount for the month. It is operational annually from May $1^{\text {st }}$ through October $31^{\text {st }}$, Monday through Friday (excluding holidays) between 11 am and $7 \mathrm{pm}$. The program is open to a variety of customers including Bundled ${ }^{5}$, Direct Access ${ }^{6}$, Community Choice Aggregation ${ }^{7}$ service and third-party aggregators. There are two types of events: day-of and day-ahead; and there are

5. Definitions of the customer varieties can be obtained from http://www.eia.doe.gov/cneaf/electricity/page/glossary.html

6. Ibid.

7. Ibid. 
two types of payments: a capacity payment for the monthly pledge and an energy payment for actual hours of reduction. Customers electing to directly sign up receive 80 percent of capacity payments and aggregators receive 100 percent of capacity payments. A participant bids five business days before the start of a month by selecting a "product" that identifies minimum and maximum hours of participation, load reduction $(\mathrm{kW})$ and energy consumption reduction for each event. Tables 2 and 3 show the possible products and the rates for each month for the dayahead and day-of programs, respectively. A participant is not required to participate more than 24 hours within a month. The incentive payments vary depending on the product selected, the month event is called, and the actual load delivered. Actual load is calculated by averaging three highest days (between $11 \mathrm{am}$ and $7 \mathrm{pm}$ ) within the last 10 admissible days prior to an event.

Table 2. Load Reduction Incentives (\$/kW) for CBP Day-Ahead Program

\begin{tabular}{|lllllrl|}
\hline Product & May & Jun & Jul & Aug & \multicolumn{1}{c|}{ Sep } & Oct \\
\hline 1 to 4 hours & 5.37 & 7.35 & 13.54 & 15.11 & 9.77 & 4.71 \\
2 to 6 hours & 5.51 & 7.54 & 14.07 & 15.63 & 10.06 & 4.81 \\
4 to 8 hours & 5.65 & 7.76 & 14.71 & 16.23 & 10.49 & 4.94 \\
\hline
\end{tabular}

Source: Lawrence Berkeley National Laboratory

Table 3. Load Reduction Incentives (\$/kW) for CBP Day-Of Program

\begin{tabular}{|lccllll|}
\hline Product & May & Jun & Jul & Aug & Sep & Oct \\
\hline 1 to 4 hours & 6.44 & 8.82 & 16.25 & 18.13 & 11.72 & 5.65 \\
2 to 6 hours & 6.61 & 9.04 & 16.89 & 18.75 & 12.07 & 5.78 \\
4 to 8 hours & 6.79 & 9.31 & 17.66 & 19.48 & 12.59 & 5.93 \\
\hline
\end{tabular}

Source: Lawrence Berkeley National Laboratory

Bidding for the CBP is done through a Web interface made available by APX. APX is a utility information system company that works with all of the IOUs in California and provides the bid collection mechanism for the CBP. APX also provides a Web page that shows the status of dayof and day-ahead programs (Figure 2). Each customer or aggregator is provided with a username and password that allows them to sign in to a website and enter their bid for the next month. APX also issues day-ahead or day-of DR event notifications for the CBP customers. Each aggregator and their customers receive notifications in the form of e-mails, pages, and/or faxes. 


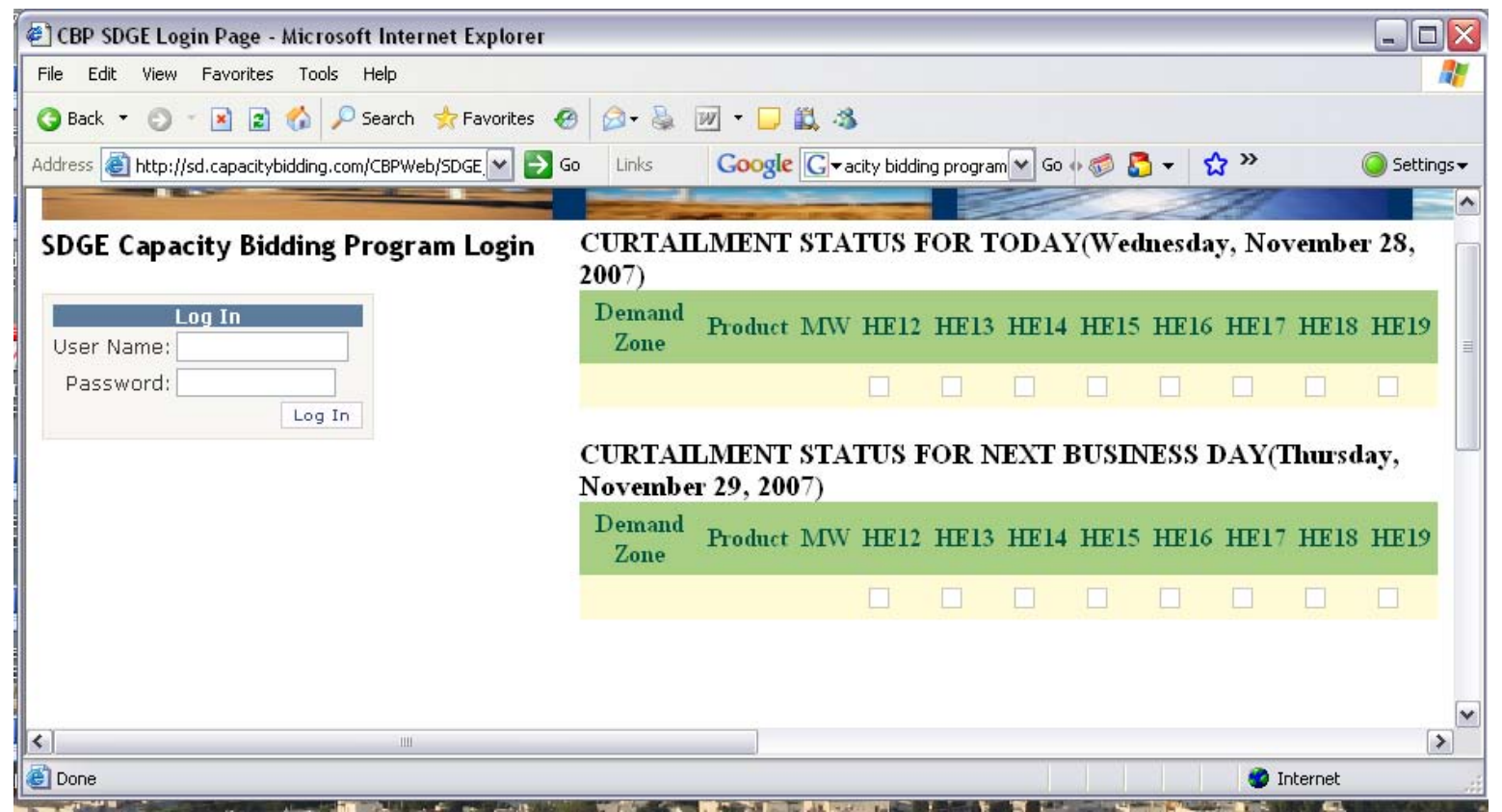

Figure 2. CBP Website from APX

Source: Lawrence Berkeley National Laboratory

The bidding page is available to the aggregators five business days before the end of a month and APX collects all the bids that are entered. Day-ahead and/or day-of notifications are issued by APX and sent to the DRAS. The notification message contains two fields: program name and product. There are six program names, each describing the day-of or day-ahead nature of the curtailment and timeframe that the curtailment is valid. These coded program names are:

1. $\mathrm{CBP} 1-4 \mathrm{DA}=1 \mathrm{pm}$ to $4 \mathrm{pm}$, Day Ahead

2. $\mathrm{CBP} 2-6 \mathrm{DA}=2 \mathrm{pm}$ to $6 \mathrm{pm}$, Day Ahead

3. $\mathrm{CBP} 4-8 \mathrm{DA}=4 \mathrm{pm}$ to $8 \mathrm{pm}$, Day Ahead

4. $\mathrm{CBP} 1-4 \mathrm{DO}=1 \mathrm{pm}$ to $4 \mathrm{pm}$, Day Of

5. $\mathrm{CBP} 2-6 \mathrm{DO}=2 \mathrm{pm}$ to $6 \mathrm{pm}$, Day Of

6. $\mathrm{CBP} 4-8 \mathrm{DO}=4 \mathrm{pm}$ to $8 \mathrm{pm}$, Day Of

The aggregator (e.g., Site Controls) developed three software clients to listen to these program names and call for curtailment to the pre-assigned group of clients. One of the clients is used for testing purposes and the other two are designated to respond to the $1 \mathrm{pm}-4 \mathrm{pm}$ period for both day-ahead and day-of programs. For the day-ahead events, the DRAS sends a "pending" signal 21 hours prior to event start time. For both day-ahead and day-of events, the DRAS sends a "moderate" signal at the beginning of an event. When the event period ends, the signal is restored to "normal" until the next event is dispatched.

Although the initial plans were to develop full integration of the DRAS with APX software systems, due to time limitations, the DRAS interface was used to initiate the automated curtailments. While the bids are requested and accepted by the APX system, the DRAS handles 
the initiation of each event for the bid period. The details of the automation system design are further discussed in Section 5 below. 


\subsection{Results}

Although the initial plans were to develop and test full Demand Response Automation Server (DRAS) integration with APX's system, due to the time limitations, the DRAS was used only for automated curtailments. Figure 3 shows how APX's and the DRAS's communications were developed. Although many alternatives for full integration of the systems were explored, due to the late start of the project, minimal system integration was preferred. APX and the DRAS were tasked with sharing the handling of monthly bids and DR events respectively. The CBP automation sequence is as follows:

1. The bidding page (Request for Bids) is available to the aggregators and aggregators are asked to submit bids five business days before the end of a month.

2. APX collects all the bids that are entered at the deadline cut off (five business days before the start of the following month).

3. Day-ahead and/or day-of notifications are issued by APX and sent to the DRAS. The DR event notification message contains three fields: program name, start (date and time) and end (date and time). There are six program names (listed in Section 4 above) each describing day-of or day-ahead nature of the curtailment and timeframe that the curtailment is valid.

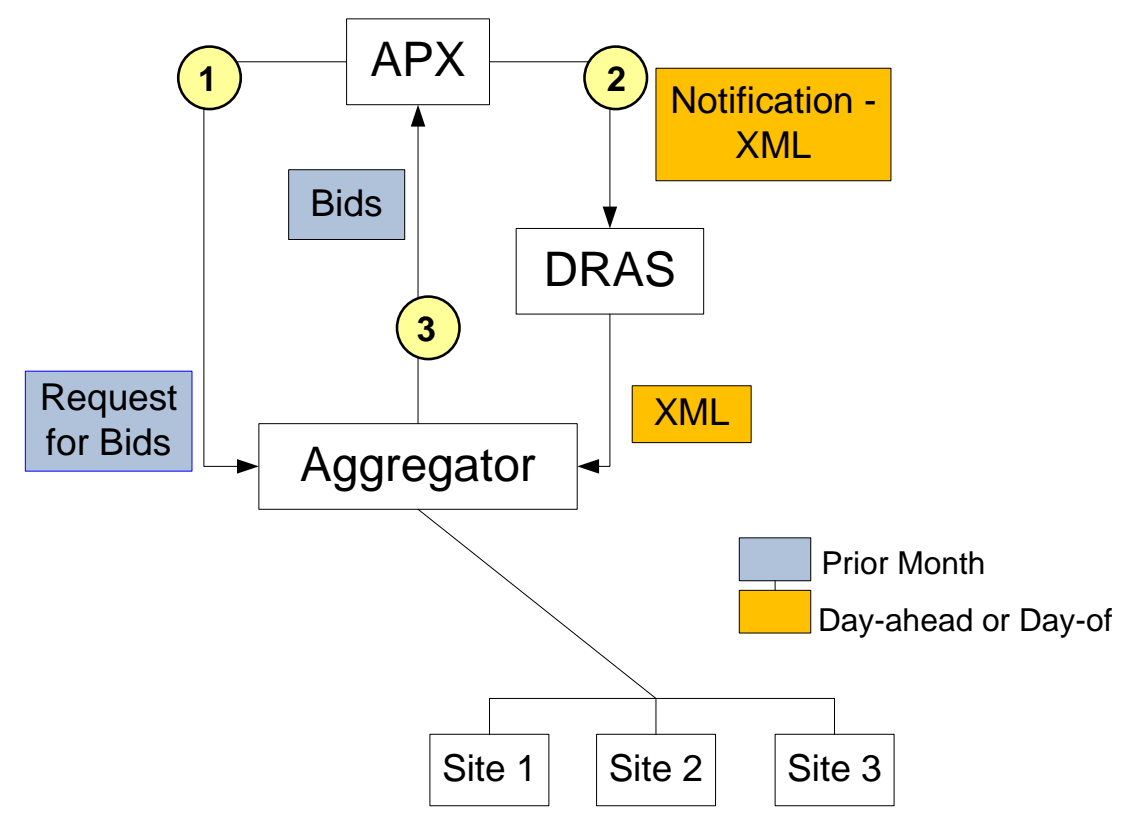

Figure 3. APX and DRAS Integration for CBP Automation

Source: Lawrence Berkeley National Laboratory

The aggregator, in this case, Site Controls, developed three software clients to listen to these program names and to call for curtailment to the pre-assigned group of clients. One of the clients is used for testing purposes and the other two are for the $1 \mathrm{pm}-4 \mathrm{pm}$ period for both 
day-ahead and day-of programs. The information is sent through the Internet as XML or Modbus ${ }^{8}$ signals.

Site Controls works with small to medium commercial buildings with loads typically below 200 $\mathrm{kW}$. Most of the sites are in the retail sector. Site Controls participated in the CBP with the following bids in 2007:

- August: 21 Meters enrolled, $205 \mathrm{~kW}$ bid, $205 \mathrm{~kW}$ delivered.

- September: 46 meters enrolled, $285 \mathrm{~kW}$ bid, $285 \mathrm{~kW}$ delivered.

- October: 62 meters enrolled, $500 \mathrm{~kW}$ bid, $500 \mathrm{~kW}$ delivered.

\subsection{End-to End Automation Test}

In October of 2007, Site Controls was ready to participate in a CBP automation test with all its sites. One DR event called on October 24, 2007 did not take place using the DR automation because the DRAS did not provide Secure Socket Layer (SSL) communication for the incoming Web services from APX. In the absence of SSL, due to security concerns, the automation was not "live" at the time the event was called. Since then, Akuacom and APX worked together to resolve this issue and SSL is now fully supported.

\subsection{DR Strategies}

Among the 62 Site Controls's facilities, typical DR strategies include pre-cooling one hour prior to an event, global space temperature set up, and lighting reductions. Most of the sites are precooled to $70 \mathrm{\circ}$ one hour prior to the start of the CBP curtailment period. The temperature is then set up about $6{ }^{\circ} \mathrm{F}$ during the curtailment period, which is typically in three- to four-hour blocks. In addition, a portion of the lights (25-50 percent depending on the site) are switched off. The communication and control system architecture between the aggregator and the DRAS is displayed in Figure 4. While bidding is manually done in the prior month through the web portal provided by APX, the DRAS triggers automated DR strategies for day-of and day-ahead events.

8. Modbus is a serial communications protocol for programmable logic controllers. 


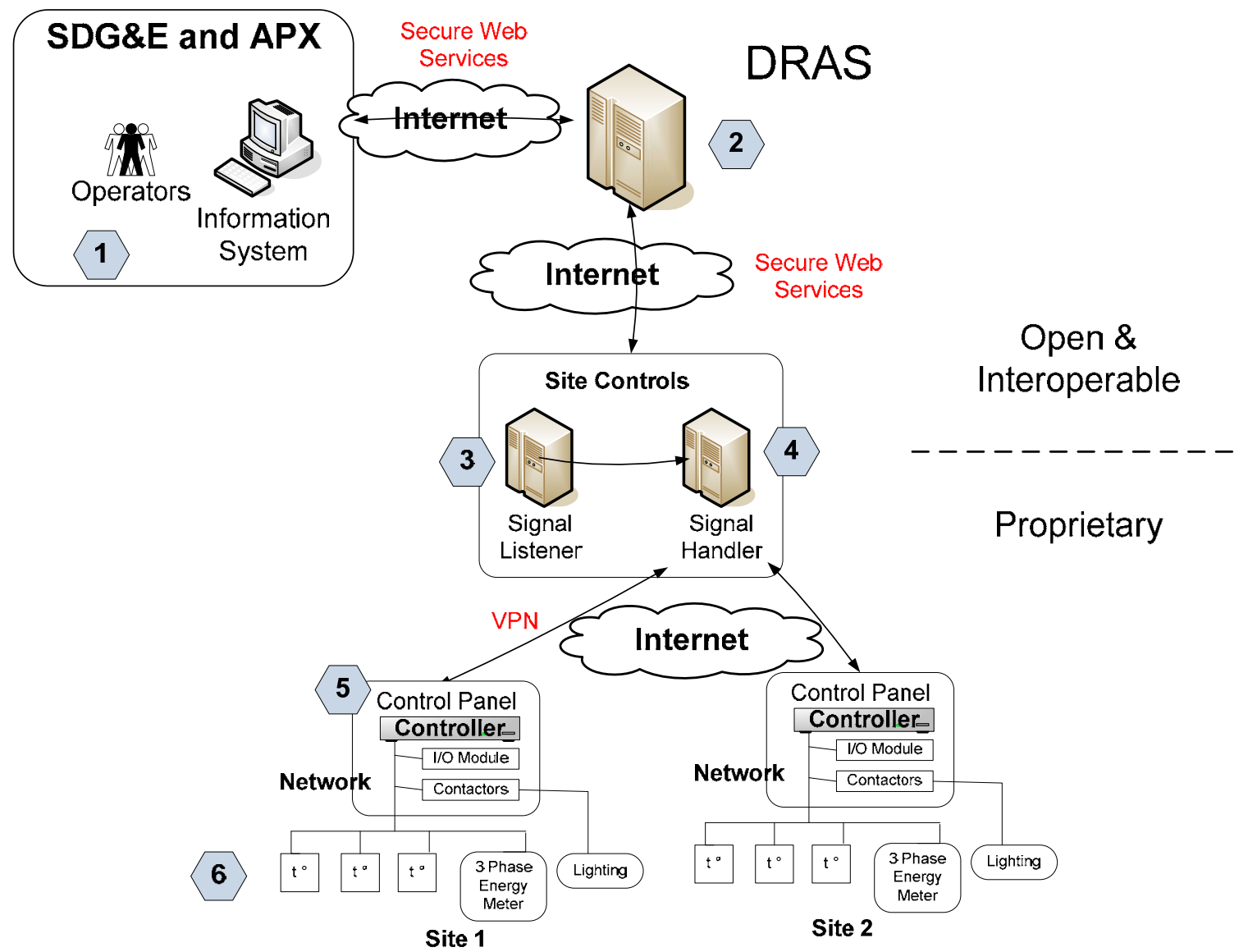

Figure 4. Open Auto-DR and Proprietary CBP Automation as Implemented by Site Controls

Source: Lawrence Berkeley National Laboratory

1. SDG\&E schedules a day-ahead or a day-of CBP event. APX sends a secure XML signal using the Open Auto-DR data model to the DRAS indicating the program name.

2. DRAS publishes the program name over secure Web services in the DRAS standard XML form. (DRAS standards have been developed in 2007 and the SDG\&E DRAS uses an earlier version of this standard communication infrastructure.)

3. Site Controls's "Listeners" poll the DRAS and receive the program names along with the priority of the signal.

4. Site Controls's "Signal Handler" forwards proprietary information to the appropriate clients out at each site via the Internet informing them of change in DR conditions.

5. Site Controls's Site Command Controller device, a Linux Box that is installed at a control panel or an electrical panel at each site, receives these signals via the Internet and sends shed commands to the thermostats and lights in terms of Modbus signals or contact relays. Site Controls's aggregators continuously monitor and control sites looking at their performance against signal requirement and assigned business rule. 
6. All loads are reduced and optimized by Site Controls's software. Demand data are captured from on-site energy meters with real-time feedback provided via aggregators. The information on DR strategies is maintained centrally by Site Controls.

Prior to the October 24, 2007 automated CBP event, the DRRC and the SDG\&E Technical Incentive test engineer tested automation of a select set of sites as part of the additional automation payment process. The tests took place on October 3, 2007. The sites that participated in the test are summarized in Table 4.

Table 4. Summary of Sites Tested on October 3, 2007

\begin{tabular}{|c|c|c|c|c|c|c|}
\hline Site & $\begin{array}{c}\text { Size } \\
\text { (sqft) } \\
\end{array}$ & $\begin{array}{l}\text { Peak } \\
(\mathbf{k W})\end{array}$ & HVAC & Pre-event & DR Strategy & Slow Recovery \\
\hline Retail C1 & 4,000 & 31 & 4 RTUs & $\begin{array}{l}\text { Pre-cool to } 70 \text { DegF } 1 \mathrm{hr} \\
\text { prior to the event }\end{array}$ & $\begin{array}{l}\text { Increase zone temperature setpoint to } 78 \text { DegF, turn } \\
\text { fans to AUTO mode, group RTUs by two's and15 } \\
\text { min. duty cycle. Turn off } 50 \% \text { of } 89 \text {-Watt fluorescent } \\
\text { fixtures and } 100 \% 32 \text {-Watt fluorescent U-tube } \\
\text { fixtures. Shut off hot water heater. }\end{array}$ & $\begin{array}{l}\text { Stagger out slowly to } \\
\text { avoid a rebound }\end{array}$ \\
\hline Retail C2 & 5,800 & 59 & 4 RTUs & $\begin{array}{l}\text { Pre-cool to } 70 \text { DegF } 1 \mathrm{hr} \\
\text { prior to the event }\end{array}$ & $\begin{array}{l}\text { Increase zone temperature setpoint to } 78 \text { DegF, turn } \\
\text { fans to AUTO mode, group RTUs by two's and15 } \\
\text { min. duty cycle. Turn off } 75 \% \text { of } 89 \text {-Watt fluorescent } \\
\text { fixtures and } 50 \% 32 \text {-Watt fluorescent U-tube } \\
\text { fixtures. Shut off hot water heater. }\end{array}$ & $\begin{array}{l}\text { Stagger out slowly to } \\
\text { avoid a rebound }\end{array}$ \\
\hline Retail C3 & 5,400 & 62 & 4 RTUs & $\begin{array}{l}\text { Pre-cool to } 70 \text { DegF } 1 \mathrm{hr} \\
\text { prior to the event }\end{array}$ & $\begin{array}{l}\text { Increase zone temperature setpoint to } 78 \text { DegF, turn } \\
\text { fans to AUTO mode, group RTUs by two's and } 15 \\
\text { min. duty cycle. Turn off } 50 \% \text { of } 89 \text {-Watt fluorescent } \\
\text { fixtures and } 100 \% 32 \text {-Watt fluorescent U-tube } \\
\text { fixtures. Shut off hot water heater. }\end{array}$ & $\begin{array}{l}\text { Stagger out slowly to } \\
\text { avoid a rebound }\end{array}$ \\
\hline Retail C4 & 5,000 & 40 & 5 RTUs & $\begin{array}{l}\text { Pre-cool to } 70 \text { DegF } 1 \mathrm{hr} \\
\text { prior to the event }\end{array}$ & $\begin{array}{l}\text { Increase zone temperature setpoint to } 78 \text { DegF, turn } \\
\text { fans to AUTO mode, group RTUs by two's and } 15 \\
\text { min. duty cycle. Turn off } 50 \% \text { of all lighting. Shut } \\
\text { off hot water heater. }\end{array}$ & $\begin{array}{l}\text { Stagger out slowly to } \\
\text { avoid a rebound }\end{array}$ \\
\hline Retail C5 & 4,436 & 10 & 4 RTUs & $\begin{array}{l}\text { Pre-cool to } 70 \mathrm{DegF} 1 \mathrm{hr} \\
\text { prior to the event }\end{array}$ & $\begin{array}{l}\text { Increase zone temperature setpoint to } 78 \text { DegF, turn } \\
\text { fans to AUTO mode, group RTUs by two's and } 15 \\
\text { min. duty cycle. Turn off } 50 \% \text { of } 89 \text {-Watt fluorescent } \\
\text { fixtures and } 100 \% 32 \text {-Watt fluorescent U-tube } \\
\text { fixtures and } 100 \% \text { halogen fixtures. Shut off hot } \\
\text { water heater. }\end{array}$ & $\begin{array}{l}\text { Stagger out slowly to } \\
\text { avoid a rebound }\end{array}$ \\
\hline Retail C6 & 23,000 & 112 & $1 \mathrm{AHU}$ & $\begin{array}{l}\text { Pre-cool to } 70 \mathrm{DegF} 1 \mathrm{hr} \\
\text { prior to the event }\end{array}$ & $\begin{array}{l}\text { Increase zone temperature setpoint to } 78 \text { DegF, } \\
\text { disable the baler, shut off hot water heater. Turn off } \\
50 \% \text { of } 59 \text {-Watt fluorescent fixtures. }\end{array}$ & No \\
\hline
\end{tabular}

Source: Lawrence Berkeley National Laboratory

A test signal was sent using the Web services client interface available through the SDG\&E DRAS instead of a test event scheduling function because at the time, the SDG\&E DRAS did not have the capability to directly schedule test events. Akuacom was made aware of this issue and it will be available in future DRAS implementations. In the absence of this feature, the test signal was initiated through the software client interface available through the DRAS at 1:04 pm by the DRRC. This time was used to ensure that Site Controls was not "waiting" for a $1 \mathrm{pm}$ command to "look" like it was automated. The XML signal was sent out in the form of a "Forced Medium Shed". While the DRAS can provide different "level" of shed commands only one level, "Medium", is used with the CBP. To evaluate the full end-to-end automation, 
the DR signal was not sent exactly at $1 \mathrm{pm}$ as was communicated with the aggregator. Instead it was sent at 1:04 pm to ensure that the aggregator software was actually "listening" and not "expecting" the automation server signal to guarantee automation.

The Site Controls's DR aggregator listens to the change in the signal and any signal above "Normal" activates the automated DR at any site that is listening to the trigger signal. It required 12 minutes for the onsite controller to receive the automation signal. Therefore, the DR strategy was activated at 1:16 pm. This was an unexpected delay due to the domain name server (DNS) issues with the aggregator software program. The delay was resolved at the end of the event when the aggregator pointed their software client to listen directly to the Internet protocol (IP) address of the DRAS instead of the DNS name provided by Akuacom.

During the test, the DR strategies implemented by the aggregator were observed and verified. The Site Controls retail stores implemented DR strategies that raise the adjusted setpoint from $74^{\circ} \mathrm{F}$ to $80^{\circ} \mathrm{F}$. In addition, half of the lights were switched off. Exact DR strategies are summarized in Appendix A. The signal to end the test was sent at 1:46 pm and the signal was received by the controller on site at $1: 53 \mathrm{pm}$.

\subsection{Demand Response Shed Analysis}

Site Controls provided customer electric interval data to the DRRC to evaluate the demand response sheds. The DRRC estimated the CBP baseline load shape for each building (Appendix A). Figure 5 shows the aggregated load shed for all of the participating stores. Table 5 summarizes the average electric shed for each store during the test period, which was 37 minutes.

The baseline calculation for the test date yielded $234.5 \mathrm{~kW}$ of peak demand for the six sites that participated in the test event. The six sites reduced their total load by $66 \mathrm{~kW}$, a 28 percent reduction from the peak. The test event was only 37 minutes and the product these sites bid into is a minimum one-hour and a maximum of four hours. While lighting load shed is sustainable over the maximum period, the sheds due to HVAC strategies are expected to diminish over time. 


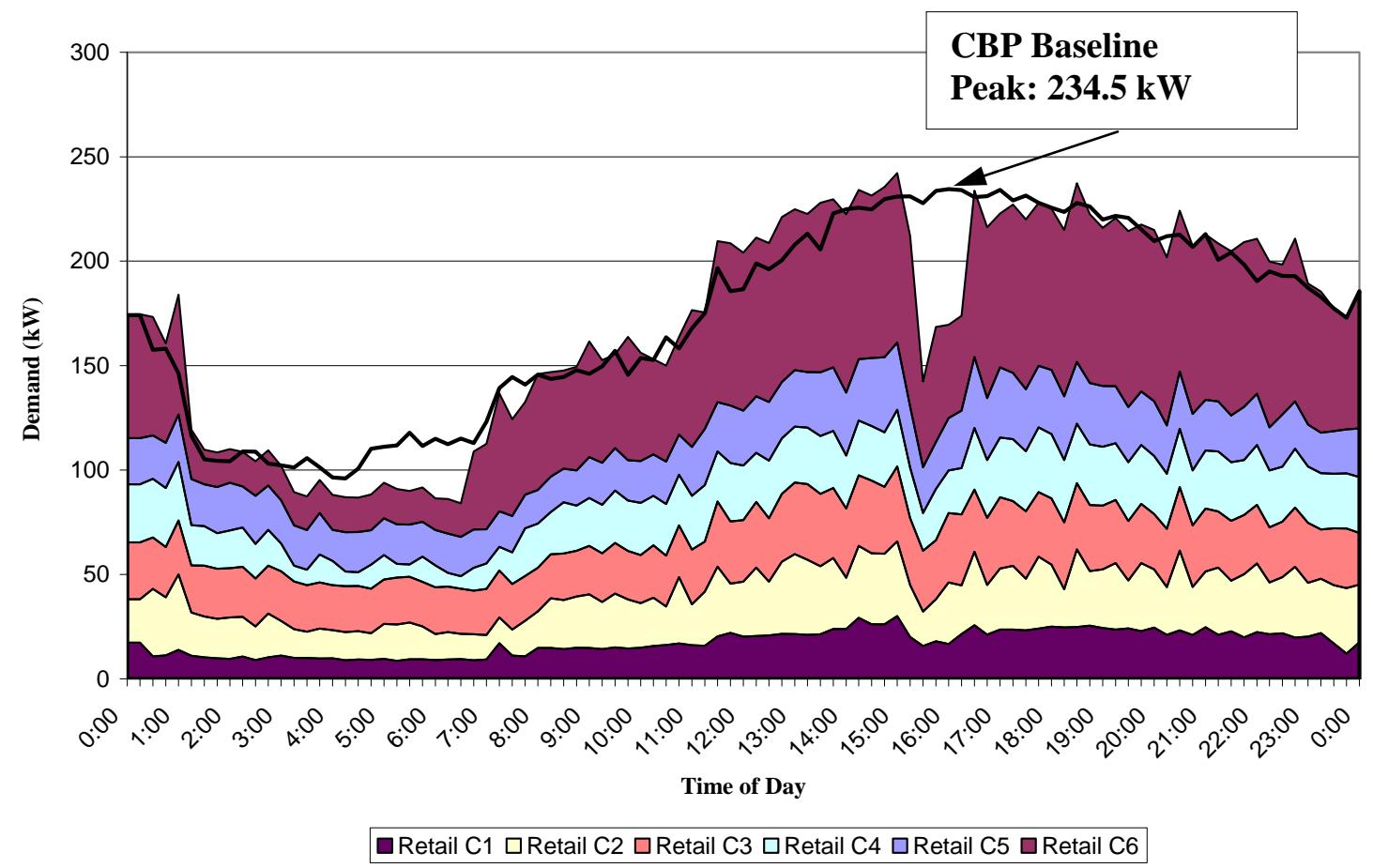

Figure 5. Aggregated Load Profile of the Test on October 3, 2007

Source: Lawrence Berkeley National Laboratory

It is notable that no significant rebound occurred after the event. A "rebound" happens after events because the systems revert back to their original settings immediately after an event and create a demand that results in a new high peak for the day and sometimes even for the month, resulting in higher demand charges.

Table 5. Summary of Average Sheds for Each Site with Total Average Shed

\begin{tabular}{|c|c|c|c|c|c|c|c|}
\hline Site & Retail C1 & Retail C2 & Retail C3 & Retail C4 & Retail C5 & Retail C6 & TOTAL \\
\hline $\begin{array}{c}\text { Average } \\
\text { Shed } \\
\text { (kW) }\end{array}$ & 6.7 & 6.6 & 0.12 & 12.6 & 6.9 & 33.1 & 66.1 \\
\hline
\end{tabular}

Source: Lawrence Berkeley National Laboratory

With the exception of Retail C3, the tests validated that the automation server executed the planned demand response strategy. Retail C3's demand during the test day prior to the test period is below the CBP baseline (see Figure 6). The lower baseline can be attributed to lower occupancy, off-line equipment due to maintenance in the store or cooler weather in that area before the test event. Lack of data for the entire summer period and not having direct contact with the onsite staff prevents the research team from understanding the reason. Since the 
baseline is lower than the actual demand through out the entire 24-hour period, the research team suggests using a morning adjusted baseline to calculate the shed for this site.

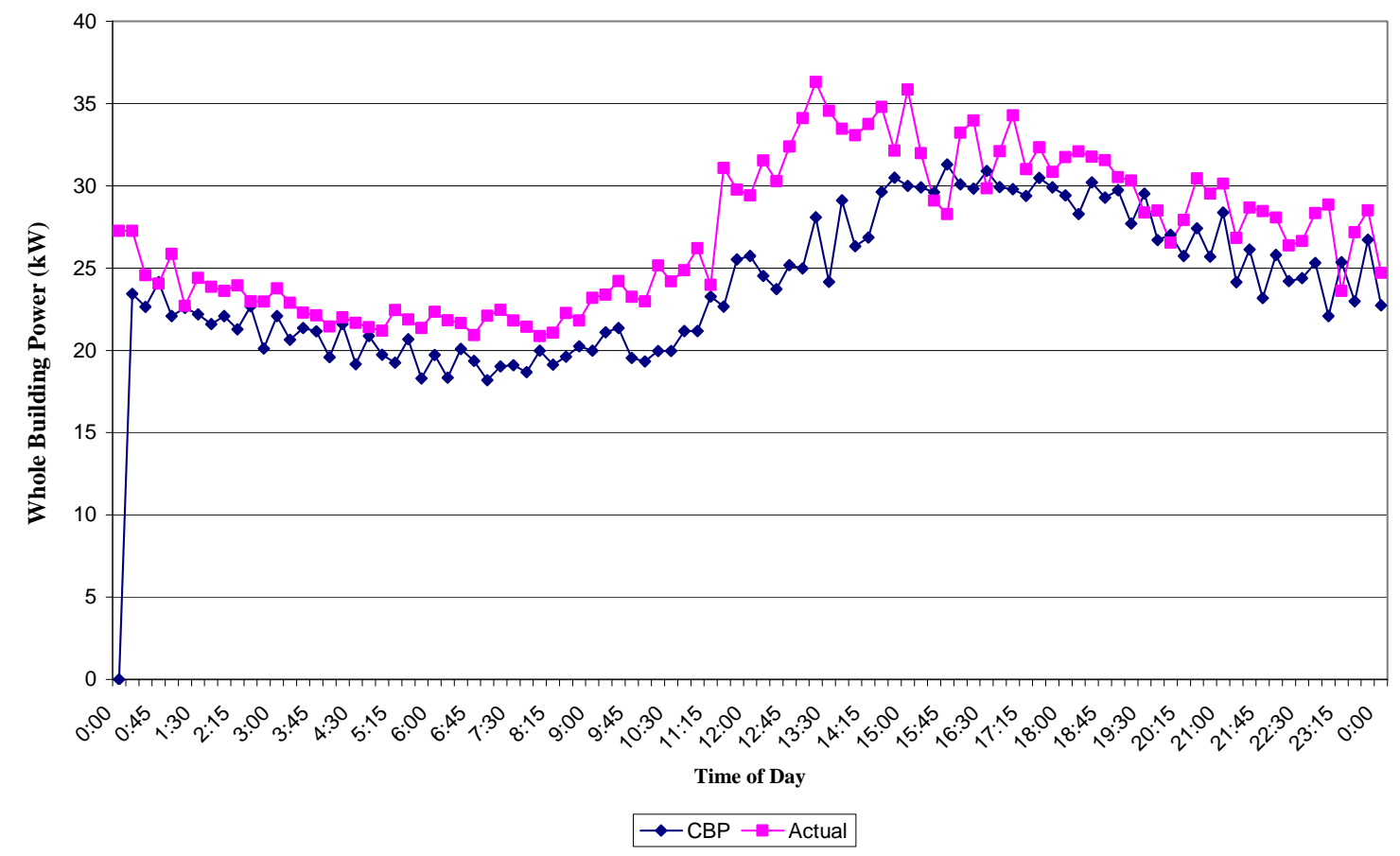

Figure 6. Retail C3 Load Profile with Its Baseline

Source: Lawrence Berkeley National Laboratory

If morning adjusted baseline is used for Retail C3, average shed amount during the test period would increase to $5.9 \mathrm{~kW}$, and the total demand savings in the test event increases to $71.8 \mathrm{~kW}$.

\subsection{Total Automated CBP Implementation Capacity}

Interviews with Site Controls suggest that they are supportive of the Open Auto-DR communications technologies because standardizing and automating the signals from utilities improves the reliability of their communications with utilities. Phone calls and emails are not ideal or reliable forms of notification. Aggregators support the idea of there being a common platform that utilities use for DR event notification, Site Controls has already equipped their entire CBP portfolio (5 customers with 77 locations and $8400 \mathrm{~kW}$ peak as of January 2008) to automated CBP. They report that they can achieve 4 megawatts (MW) of DR from these 8.4 MW of total demand with an approximate 50 percent shed for the one- to four-hour duration period. 


\subsection{Discussion and Recommendations}

This section summarizes the key issues, recommendation and findings in three main categories:

1. Enabling the Capacity Bidding Program (CBP) Automation

2. Aggregator Participation

3. Program Development

\subsection{Enabling CBP Automation}

The automation of CBP was the first time Open Automated Demand Response (Open Auto-DR) was tested with an aggregator technology. This implementation required the demand response automation server (DRAS) to be integrated into an existing program with various partners, including San Diego Gas and Electric (SDG\&E) Technical Audit/Technology Incentives (TA/TI) Program, APX, and Site Controls. Issues related with technology implementation and integration are summarized below:

- Fast DRAS development for Automated CBP (AutoCBP), integration with APX and Site Controls. Akuacom was able to develop and customize the automation program in four weeks during the CBP season. Separation of monthly bidding from CBP event notification throughout the month and allowing these to be handled by different systems resulted in less integration effort, thus shortening the time it took to implement AutoCBP.

- DRAS software client was easy to implement. - LBNL sent a client template to several aggregators two days before SDG\&E's Technical Workshop with Energy Services Companies organized by SDG\&E. Five out of six aggregators were able to develop a software client and establish a connection with the DRRC's research DRAS. There is a wide range of technologies used among DR aggregators. Those that utilize web services to optimize demand reduction are better candidates to use the Automated Demand Response (AutoDR) systems. Interviews with Site Controls suggest that they are supportive of the AutoDR communications technologies and plan to move their entire CBP portfolio to automated CBP.

- Site Controls developed a software client within a short amount of time. Since their communication infrastructure is very similar to the one being used by DRAS and its clients, the software developer was able to develop their clients in a few days. Site Controls even enhanced the software client template that was made available to them and offered to share it with Akuacom and other aggregators.

\subsection{Aggregator Participation}

The CBP automation test event demonstrated that the automation functions as intended and that the sheds took place when the signal was received. However, there were a few notable issues. 
- Size of automated load: A CBP event was called after the completion of the automation work on October 24, 2007 due to fires in San Diego area. This event was not automated because the system was not "live" due to a security issue that was outstanding at that time. On the same day, Site Controls bid 500 kilowatts $(\mathrm{kW})$ for their facilities in the CBP and delivered over $600 \mathrm{~kW}$ of demand reduction. If the automation system were active, all of Site Control's facilities that participated in the event would have participated automatically.

- Signal delay: As stated earlier, there was a significant delay, (about 10 to 12 minutes) between the time the signal was issued and the time when it was received by the on-site controller. After the test event, the aggregator changed the DRAS configuration files to make the client listen to an actual Internet protocol (IP) address rather than a domain name server (DNS) and the signals were almost instantaneous. This is an outstanding automaton issue that can be resolved if the aggregator modifies their software client. This modification could be evaluated with another end-to-end test.

- Duration of DR event: In previous Open Auto-DR tests, the duration of the event has been a minimum of three hours. If the purpose of a test is to demonstrate automation capability, short tests that demonstrate this are sufficient. If the purpose of the test is to evaluate the size of the demand response, the tests should be at least two hours in duration. HVAC zone temperature adjustment strategies have both transient and steady state savings. In short tests, the transient savings dominate and over time, its impact on demand savings decrease as the temperatures in the zones increase. The research team recommends that if automation is being tested at the same time with the load shed test, for any temperature adjustment strategy, the test event should be at least two hours long. This however is not required for lighting strategies that use switching or direct load control strategies.

- Aggregators can participate in Automated DR programs. CBP automation experience showed that aggregators' systems can listen to standard signals initiated by utilities and deliver it to their customers without human involvement. In most cases, $\$ 250 / \mathrm{kW}$ covers the cost of these installations.

\subsection{Program Development}

As this implementation experience shows, the aggregators are able and willing to participate in automated programs. There is a need to design DR programs and TA/TI procedures that include processes for automation. This section summarizes the program-related observations.

- Deployment of Open Auto-DR program with aggregators differs from previous priceresponse Open Auto-DR implementation models. The automated price responsive programs in the other investor-owned utility territories (i.e., PG\&E and SCE) have been adopting a model where a third-party program management group directly recruits the customers, trains, and certifies control vendors to assist customers with automation, and oversee TI payments. In SDG\&E's CBP automation, aggregators take over these activities. They recruit customers, installing and maintaining the controls and communication infrastructure that enables automation. In addition, from a customer's 
perspective, while aggregators directly control their equipment by sending the actual temperature setting or light switching signal in real time during a CBP event, price responsive programs that are enabled send a level of shed to trigger pre-programmed strategies. Therefore there is no variation in strategies for each event for automated price-responsive programs. However, there may be variations in conditions at a customer's site depending on the shed amount obtained from the entire portfolio for programs with aggregator involvement.

- Current structure of SDG\&E's TA/TI program is able to accommodate Open Auto-DR enablement. The TA/TI program requires each customer to go through a technical audit and requests the program support engineer to fill out a technology incentives form. After the form is completed and the equipment is installed, the same engineer visits the site to oversee DR strategies being implemented and demand reduction specified by the form takes place. The aggregator calls for the test event through their automation system. Automation can also be tested at the same time if the test engineer is trained in calling test events through the DRAS and the TI form templates are changed to include this information.

- Process development and training for 2008 program needed for successful continuation of the program. A number of tasks that the DRRC performed in 2007 should be transferred to SDG\&E. These tasks include: DRAS operation, technical support, and field tests. In addition, SDG\&E should change some of their processes to incorporate automation installation and inspection. A set of guidelines that describe end-to-end automation can assist aggregators in deciding whether their technology is suitable for Open Auto-DR. 


\subsection{Summary and Conclusions}

This report has discussed the collaboration between the Demand Response Research Center (DRRC) and San Diego Gas and Electric's (SDG\&E's) program, Akuacom, APX, and Site Controls to develop and deploy a fully automated Capacity Bidding Program (CBP). The goals of the project were to develop and demonstrate an automated demand response (DR) program that involved the participation of aggregators; to explore the role of automation in increasing participation rates; and to explore the controls infrastructure and strategies that are being used by small commercial facilities. To achieve the objectives, the research had four key elements:

1. Interview aggregators to identify sites that can deliver end-to-end automation.

2. Design and build the Automated CBP by developing requirements and integration concepts.

3. Test the automation between all the parties involved.

4. Field test at selected facilities that deploy the same technology of the selected aggregator.

This project successfully achieved the objective to develop and demonstrate how an open, webbased interoperable automated notification system for capacity bidding can be used by aggregators for DR. The system was effective in initiating a fully automated DR shed at the aggregated sites. This project has demonstrated how aggregators can integrate their DR automation systems with the DR Automation Server and CBP. The research team developed an example of a method to characterize aggregator's automation systems. The communications systems from the aggregator to the end-use controls are proprietary signals and are not part of an open, interoperable system. However, the characterization of the infrastructure suggests that a fully automated system is being used.

The DRRC will continue to conduct research to facilitate broader implementation of Auto-DR systems in commercial and industrial facilities. The long-term vision is to embed the automation clients and DR shed modes into new construction by including them in building codes. There is a need to educate building operators, controls engineers, and control companies on the concepts of DR and the opportunities with automation. As utilities, independent system operators, and energy service providers become more familiar with DR, moving towards automated DR will help improve making the demand-side reductions more reliable and repeatable load reductions from customers. Facility operators and engineers will turn to their controls vendors to deliver additional capabilities to the existing energy management and control systems. The DRRC is working with the American Society of Heating, Refrigerating and Air-Conditioning Engineers, National Institute of Standards and Technologies (NIST) and other organizations to build a de facto standard for DR event propagation based on the communication foundation of Auto-DR. Work is underway to develop an open standards based BACnet ${ }^{9}$ Web Services client that works with the proposed DR communication infrastructure for implementation in energy management systems. This effort is part of a broader plan to evaluate the feasibility of requiring this communications capability into new

9. A Data Communications Protocol for building Automation and Controls Networks. 
commercial buildings through California's building energy code (Title 24) in 2011. Efforts to embed automated DR clients in buildings will facilitate DR for aggregators as well as improve the potential for buildings to be price responsive. 


\subsection{References}

California Public Resources Code, Section 25402, 2005. Title 24 - California's Energy Efficiency Standards for Residential and Non-residential Buildings.

Coughlin, K., M. A. Piette, C. Goldman and S. Kiliccote. Estimating Demand Response Load Impacts: Evaluation of Baseline Load Models for Non-Residential Building in California. Demand Response Research Center, Lawrence Berkeley National Laboratory. LBNL-63728. January 2008.

Motegi, N., M.A. Piette, D.Watson, S., Kiliccote, P. Xu,. Introduction to Commercial Building Control Strategies and Techniques for Demand Response. LBNL Report 59975. May 2007. Available at drrc.lbl.gov.

Piette, M.A., O. Sezgen, D.S. Watson, N. Motegi, and C. Shockman. 2005a. Development and Evaluation of Fully Automated Demand Response in Large Facilities. Lawrence Berkeley National Laboratory CEC-500-2005-013. LBNL-55085. Berkeley CA, January.

Piette, M.A., D.S. Watson, N. Motegi, N. Bourassa, and C. Shockman. 2005b. Findings from the 2004 Fully Automated Demand Response Tests in Large Facilities. Lawrence Berkeley National Laboratory. CEC-500-03-026. LBNL-58178. Berkeley CA, September. Available at drrc.lbl.gov.

Piette, M. A., D. Watson, N. Motegi, S. Kiliccote, P. Xu (Lawrence Berkeley National Laboratory). Automated Critical Peak Pricing Field Tests: Program Description and Results. Report to the Pacific Gas and Electric Company Emerging Technologies Program and California Institute for Energy and the Environment. LBNL-59351. April 2006.

Piette, M.A., D.Watson, N. Motegi, and S., Kiliccote. Automated Critical Peak Pricing Field Tests: 2006 Pilot Program Description and Results. LBNL Report 62218. May 2007.

Piette, M.A., S., Kiliccote and G., Ghatikar. Design and Implementation of an Open, Interoperable Automated Demand Response Infrastructure. Grid Interop Forum. November 2007.

Standard 135--2004--BACnet-A Data Communication Protocol for Building Automation and Control Networks. ANSI approved. Published by ASHRAE. 2004. ISBN/ISSN: 1041-2336 


\subsection{Glossary}

BACnet

CBP

CLIR

CPP

DNS

DR

DRAS

DRRC

EIS

EMCS

EPRI

HVAC

IOU

IP

$\mathrm{kW}$

LBNL

MW

NIST

OAT

Open Auto-DR

PG\&E

SCE

SDG\&E

SOA

SSL

TA/TI

TAG

VPN

XML
Data Communication Protocol for Building Automation and Control Networks, American Society of Heating, Refrigerating and Air-

Conditioning Engineers Standing Standard Project Committee 135

Capacity Bidding Program

Client and Logic with Integrated Relay

Critical Peak Pricing

Domain Name Server

Demand Response

Demand Response Automation Server

Demand Response Research Center

Energy Information Systems

Energy Management and Control Systems

Electric Power Research Institute

Heating, Ventilation and Air Conditioning

Investor-owned utility

Internet protocol

Kilowatts

Lawrence Berkley National Laboratory

Megawatt

National Institute of Standards and Technologies

Outside Air Temperature

Open, Non-Proprietary Automated Demand Response

Pacific Gas and Electric Company

Southern California Edison

San Diego Gas \& Electric Company

Service Oriented Architecture

Secure Socket Layer

Technical Audit/Technology Incentives

Technical advisory group

Virtual Private Network

Extensible Mark-up Language 